\title{
Concurrent Arterial and Venous Thrombosis in a Patient with Ulcerative Colitis in Remission Phase
}

\author{
Hamid Ali Kalwar, Tauqeer Shaikh and Muhammad Hussain Baloch \\ Department of Gastroenterology, Liaquat National Hospital \& Medical College, Karachi, Pakistan
}

\begin{abstract}
Vascular thrombosis is an unusual yet well-known extra-intestinal complication of ulcerative colitis, usually involving the peripheral veins and occasionally the cerebral veins and the arterial system. We, herein, report a case of a 45-year female with ulcerative colitis in remitting phase presenting with anemia and right lower limb swelling and discoloration due to extensive arterial and venous thrombosis. Early and accurate diagnosis of thrombotic complications is essential to start limb-saving and lifesaving therapy.
\end{abstract}

Key Words: Thrombotic, Ulcerative colitis.

How to cite this article: Kalwar HA, Shaikh T, Baloch MH. Concurrent Arterial and Venous Thrombosis in a Patient with Ulcerative Colitis in Remission Phase. J Coll Physicians Surg Pak 2021; 31(02):215-217.

\section{INTRODUCTION}

Around $25 \%$ to $36 \%$ patients of idiopathic inflammatory bowel disease (IBD) are reported to present withextra-intestinalcomplications. ${ }^{1}$ The common extra-intestinal manifestations are peripheral arthritis (10.7\%) sacroilitis (14\%). Although vascular (2\%), mucocutaneous (2.7\%) and ocular (8\%) complications are rare. ${ }^{2}$ More than $60 \%$ of the vascular complications are accounted for by peripheral venous thrombosis or pulmonary embolism. Peculiar sites of thrombosis involve portal, mesenteric and cerebral veins. Arterial thrombosis is extremely uncommon. It has been proposed that thromboembolic events are more common in young patients with active and extensive disease. It occurs often in unusual sites and is associated with high morbidity and mortality. ${ }^{3}$

We report a young woman presenting with stable disease of ulcerative colitis, coupled with thrombosis, including the peripheral venous and arterial system, all occurring in the course of the same episode of illness.

\section{CASE REPORT}

A 45-year female, presented to Outpatient Department (OPD) with complaints of dyspnea, palpitation, easy fatiguability accompanied with swelling, pain and blackish discoloration in the right lower limb for one and a half months (Figure 1). She was a follow-up case of ulcerative colitis, on mesalamine and deltacortil, now in remission phase on same medications.

Correspondence to: Dr. Hamid Ali Kalwar, Department of Gastroenterology, Liaquat National Hospital \& Medical College, Karachi, Pakistan

E-mail: hamidgulk@yahoo.com

Received: January 09, 2019; Revised: December 14, 2019; Accepted: January 07, 2020

DOI: https://doi.org/10.29271/jcpsp.2021.02.215

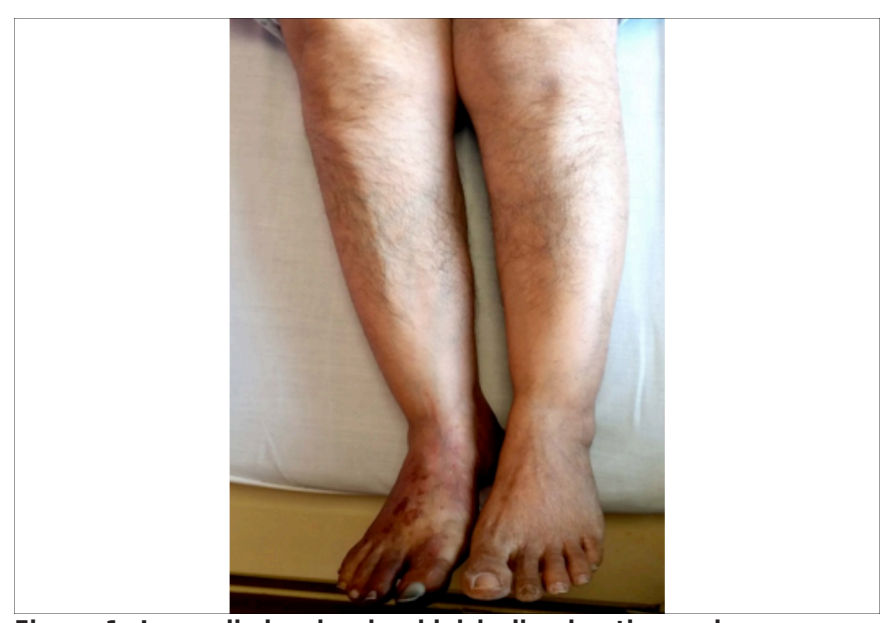

Figure 1: Lower limbs showing bluish discoloration and gangrenous changes in right lower limb.

There was no any complain of diarrhea, fever, blood in stools, decreased urine output, prolonged immobilisation, oral contraceptive pill intake, trauma, chest pain, air travel, stroke or recurrent miscarriages. Recently, she had restricted mobility because of pain, swelling of right lower limb, dyspnea, and easy fatigability. On inspection, patient was conscious and oriented to place, time and person having a pulse rate of $88 /$ minute in three limbs, while pulses were feeble in right lower limb. Right brachial artery had blood pressure of 100/70 mm Hg. Right leg and foot were warm, non-tender, and swollen with bluishblackish discoloration and the distal pulses were feeble. Central nervous system (CNS) examination revealed patient as conscious, and oriented to place, time and person. There was no any sensory or motor deficit. All reflexes were normal. The cardiovascular, abdominal, and respiratory systems were normal.

During hospitalisation, two packed cells were transfused, and vascular surgery was taken on board who advised ultrasound 
(US) arteriovenous Doppler (Figure 2) and CT-angiogram of right leg (Figure 3), which showed both arterial and venous thrombosis.

Limb salvage surgery was planned. Right leg arterectomy with embolectomy was done. During surgery, one more packed cell unit was transfused. Patient tolerated the procedure well and was discharged on direct oral anticoagulants (rivaroxaban), 15 mg per oral, twice daily for 21 days; then 20 mg per oral daily for whole life. Patient was well and stable on follow-up visit after a suitable interval.

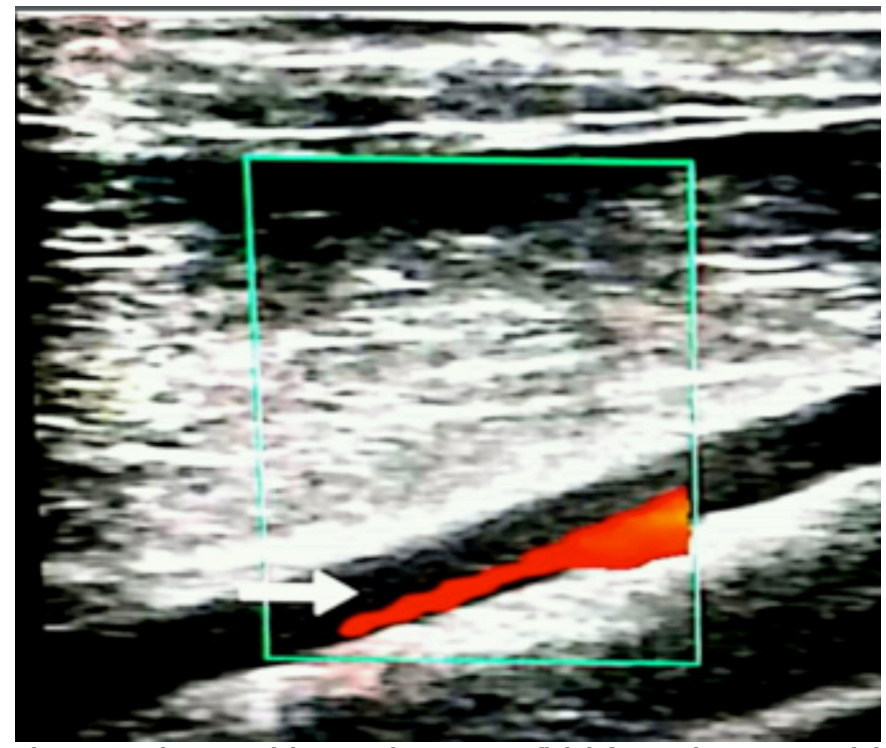

Figure 2: Ultrasound image shows superficial femoral artery partial thrombus on colour Dopplerimage.

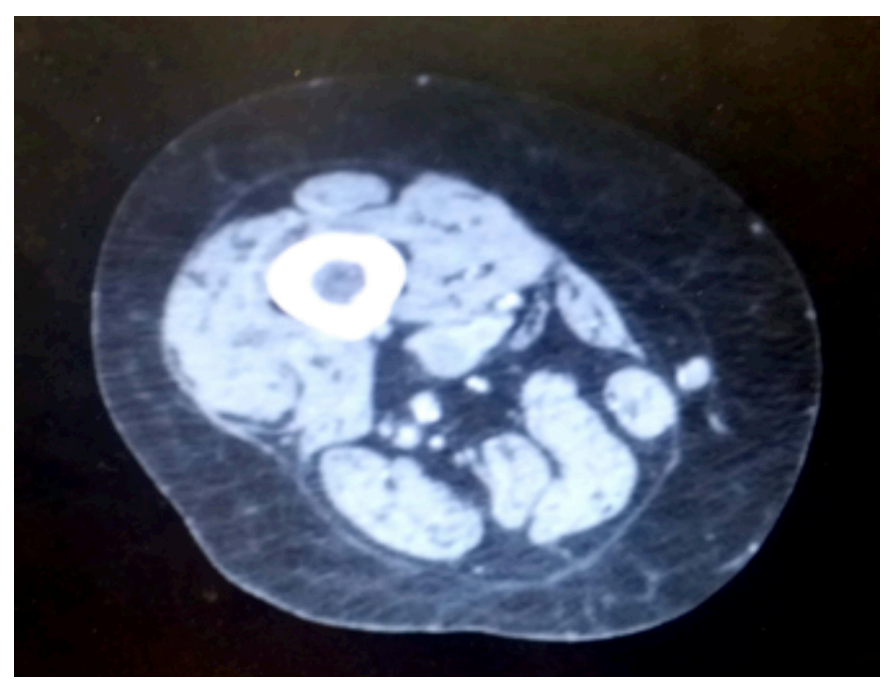

Figure 3: Axial image, arterial phase, shows incomplete non opacification of superficial femoral artery consistent with partial thrombus.

\section{DISCUSSION}

Increased coagulability is an unusual, yet well-known feature of ulcerative colitis. In 1936, Bargen and Barker published the first ever report of thromboembolic phenomena complicating IBD. ${ }^{4}$ Since then, several studies have highlighted this association. Talbot et al. reported that thromboembolic complications occurred in $1.3 \%$ of patients with IBD over a period of 11 years, ${ }^{5}$ while pulmonary embolism or deep vein thrombosis (DVT) was found in almost $66 \%$ of them with a mortality rate as high as $25 \%$.

Patients with IBD are found to bethreetimes more prone tothromboembolic complications than controls and approximately the risk transcends to 15 times during flares. ${ }^{6}$ While the precise pathogenesis remains unknown, IBD has been associated with occurrence of increased arterial and venous thrombosis. ${ }^{7}$

Occurrence of peripheral arterial and venous thrombosis together, in patients with relapse of IBD, remains uncommon despite both being relatively common. Ultrasonography with Doppler can assist in screening patients for these complications. Due to prompt results and easy availability, CT-angiography remains the investigation of choice to confirm the diagnosis.

Treating such patients is still a matter of concern due to very high morbidity and mortality associated in such cases, if immediate treatment is not started. Heparin use is rationalised in IBD with thrombosis, despite not being indicated as a preventive measure. The optimal duration of anticoagulation remains unknown as the pathogenesis is still not clear.

A European cohort study evaluated that afterstopping of anticoagulant therapy, the five years risk of recurrence goes up to $33 \%$. Moreover, with a first unprovoked venous thromboembolism (VTE), the threat of IBD recurrence escalated to 2.5 -fold. This implies that IBD is a continuing and endless threat for recurrent VTE; and consequently supports an indefinite anticoagulant therapy for the victims of IBD having VTE. ${ }^{8}$ However, the risk-benefit ratio must be evaluated. Lately, the Canadian Society of Gastroenterology has suggested the usage of anticoagulation for proven thrombotic events, and for prophylaxis of thrombosis in patients for surgery. ${ }^{9}$ However, randomised-controlled trials are needed to justify this recommendation.

Inflammatory activity, pregnancy, surgery, repeated hospitalisation, disease type (e.g. extensive colonic involvement, fistulising disease) and treatment (mostly steroids) with stimulation of the coagulation system, impairment of fibrinolysis, down regulation of natural anticoagulant mechanisms, dysfunction of the endothelium and increased platelet count and reactivity have been implicated in the occurrence of thrombotic cases in these patients. ${ }^{10}$ Recent literature specify few stably remitted patients of IBD with case reports of thrombosis. Such a case of venous thrombosis was recently reported in a remitted child. ${ }^{11}$ Clinicians must be vigilant of such consequences and take satisfactory prophylactic means in patients who are undergoing treatment, and hospitalised or/and submitted to surgery.

\section{PATIENT'S CONSENT:}

Informed verbal consent was taken from the patient.

\section{CONFLICT OF INTEREST:}

Authors declared no conflict of interest.

\section{AUTHORS' CONTRIBUTION:}

HAK: Did manuscript writing, editing and proofreading.

TS, MHB: Did data collection and manuscript writing. 


\section{REFERENCES}

1. Danzi JT. Extraintestinal manifestations of idiopathic inflammatory bowel disease. Arch Intern Med 1988; 148: 297-302. doi:10.1001/archinte.1988.00380020041008.

2. Kochhar R, Mehta SK, Nagi B, Bhatia V, Goenka MK, Malik AK, et al. Extraintestinal manifestation of idiopathic ulcerative colitis. Ind J Gastroenterol 1991; 10(3):88-9.

3. Jackson LM, O'Gorman PJ, O'Connell J. Thrombosis in inflammatory bowel disease: Clinical setting, procoagulant profile and factor V Leiden. QJM 1997; 90(3):183-8. doi.org/ 10.1093/qjmed/90.3.183.

4. Bargen JA, Barker NW. Extensive arterial and venous thrombosis complicating chronic ulcerative colitis. Arch Intern Med 1936; 58(1):17-31. doi:10.1001/archinte.1936. 00170110025002.

5. Talbot RW, Heppell J, Dozois RR, Beart RW. Vascular complications of inflammatory bowel disease. Mayo Clin Proc 1986; 61(2):140-5. doi:10.1016/S0025-6196(12)65200-8.

6. Ashrafi MR, Hosseini F, Alizadeh H, Sani MN. Pseudotumor cerebri in a case of ulcerative colitis with sagittal sinus thrombosis. Iran J Pediatr 2013; 23(1):109-12.

7. Zezos P, Kouklakis G, Saibil F. Inflammatory bowel disease and thromboembolism. World J Gastroenterol 2014; 20(38):13863-78. doi: 10.3748/wjg.v20.i38.13863.

8. Jain S, Bhatt P, Muralikrishna GK, Malhotra P, Kumari S, Varma $\mathrm{S}$, et al. Extensive arterial and venous thrombosis in a patient with ulcerative colitis: A Case Report. Med Gen Med 2005; 7(2):10.

9. Bernstein CN, Bitton A, Chan AK, Griffiths AM, Leontiadis GI. Consensus statements on the risk, prevention, and treatment of venous thromboembolism in inflammatory bowel disease: Canadian association of gastroenterology. Gastroenterol 2014; 146:835-48. doi:10.1053/j.gastro. 2014.01.042.

10. Magro F, Soares JB, Fernandes D. Venous thrombosis and prothrombotic factors in inflammatory bowel disease. World J Gastroenterol 2014; 20(17):4857-72. doi: 10.3748/ wjg.v20.i17.4857.

11. Gysemans W, Van Geet C, Janssens E, Alliet P. Venous thrombosis in a child with ulcerative colitis in remission: $A$ case report. Acta Gastroenterol Belg 2014; 77(1):71-74. 